\title{
On Fredholm properties of Toeplitz operators in Bergman spaces
}

Article

Accepted Version

Taskinen, J. and Virtanen, J. (2020) On Fredholm properties of Toeplitz operators in Bergman spaces. Mathematical Methods in the Applied Sciences, 43 (16). pp. 9405-9415. ISSN 01704214 doi: https://doi.org/10.1002/mma.6268 Available at https://centaur.reading.ac.uk/88610/

It is advisable to refer to the publisher's version if you intend to cite from the work. See Guidance on citing.

To link to this article DOI: http://dx.doi.org/10.1002/mma.6268

Publisher: Wiley

All outputs in CentAUR are protected by Intellectual Property Rights law, including copyright law. Copyright and IPR is retained by the creators or other copyright holders. Terms and conditions for use of this material are defined in the End User Agreement.

\section{www.reading.ac.uk/centaur}

\section{CentAUR}

Central Archive at the University of Reading

Reading's research outputs online 


\title{
ON FREDHOLM PROPERTIES OF TOEPLITZ OPERATORS IN BERGMAN SPACES
}

\author{
JARI TASKINEN AND JANI VIRTANEN
}

\begin{abstract}
We consider Toepliz operators with integrable symbols acting on Bergman spaces $A^{p}, 1<p<\infty$, of the open unit disc of the complex plane. We combine some of the best known results on compactness of Toeplitz and Hankel operators in order to generalize the results on Fredholm properties of Toeplitz operators. We pay special attention to some concrete examples.
\end{abstract}

\section{INTRODUCTION AND PRELIMINARIES.}

The theory of Toeplitz operators in Bergman spaces have found applications in areas like deformation quantization of theoretical physics, which relates to geometric structures of classical mechanics their algebraic quantum counterparts, see [1], [2], and to soliton theory, [3], [4], where these operator theoretic methods can be applied to relax the standard assumptions on initial conditions of the KdV-equation. A Toeplitz operator $T_{a}$ is determined by its symbol $a$, which is usually an integrable function on the underlying analytic manifold $\Omega$. However, even in the simplest case when $\Omega$ is the unit disc $\mathbb{D}$ of the complex plane $\mathbb{C}$, not all operator theoretic properties of $T_{a}$ following from the properties of the symbol are adequately understood: apparently, unnecessarily strong a priori assumptions on the regularity of $a$ have been needed for many existing resuls.

In this paper we concentrate on relaxing the conditions for the Fredholm property of the operator $T_{a}$. Recall that a bounded operator $T$ in a Banach space $X$ is Fredholm, if it has an inverse $S$ modulo compact operators, i.e. the $T S-I$ and $S T-I$ are compact in $X$. (We recall the details of the definitions and basic statements at the end of this section.) As well known, the Fredholm property is crucial in the theory of solutions of integral equations, which is in fact historically the starting point to the study of this operator class.

Consider the Banach space $L^{p}:=\left(L^{p}(\mathbb{D}, d A),\|\cdot\|_{p}\right)$, where $1<p<\infty$ and $d A=\pi^{-1} r d r d \theta$ is the normalized area measure on $\mathbb{D}$, and denote the Bergman space by $A^{p}$, which is the closed subspace of $L^{p}$ consisting of analytic functions. The Bergman projection $P$ is the orthogonal projection of $L^{2}$ onto $A^{2}$, and it has the integral representation

$$
\operatorname{Pf}(z)=\int_{\mathbb{D}} \frac{f(\zeta)}{(1-z \bar{\zeta})^{2}} d A(\zeta)
$$

2000 Mathematics Subject Classification. 47B35.

Key words and phrases. Toeplitz operator, Hankel operator, Fredholm operator, Bergman space, compact operator.

JT was supported by a research grant from the Faculty of Science of the University of Helsinki. JV was supported by Engineering and Physical Sciences Research Council (EPSRC) grants $\mathrm{EP} / \mathrm{M} 024784 / 1$ and $\mathrm{EP} / \mathrm{T} 008636 / 1$. 
for $z \in \mathbb{D}$. It is also known to be a bounded projection of $L^{p}$ onto $A^{p}$ for every $1<p<\infty$. We denote the complementary projection of $P$ by $Q:=I-P$, where $I$ is the identity operator.

For an integrable function $a: \mathbb{D} \rightarrow \mathbb{C}$ and, say, bounded analytic functions $f$, the Toeplitz operator $T_{a}$ with symbol $a$ is defined by

$$
T_{a} f(z)=P(a f)(z)=\int_{\mathbb{D}} \frac{a(\zeta) f(\zeta)}{(1-z \bar{\zeta})^{2}} d A(\zeta)
$$

for $z \in \mathbb{D}$. By posing suitable sufficient conditions on the symbol $a$, the operator $T_{a}$ extends as a bounded operator $A^{p} \rightarrow A^{p}$, see e.g. [5], [6]. This is trivially true for bounded $a$. The corresponding Hankel operator $H_{a}: A^{p} \rightarrow L^{p}$ is defined by $H_{a}=M_{a}-T_{a}$, where $M_{a}: A^{p} \rightarrow L^{p}$ is the pointwise multiplier $M_{a}: f \mapsto a f$. Hence, we have

$$
H_{b} f(z)=\int_{\mathbb{D}} \frac{(b(z)-b(\zeta)) f(\zeta)}{(1-z \bar{\zeta})^{2}} d A(\zeta)
$$

for $z \in \mathbb{D}$.

In this paper we deal with the Fredholm properties of Toeplitz operators $T_{a}: A^{p} \rightarrow$ $A^{p}$ both theoretically and by means of concrete examples. In Theorem 2.2, which is our main result, we give a sufficient condition for $T_{a}$ to be Fredholm for a wide class of symbols $a$ in $L^{1}$. While the proof is in a sense rather straightforward, the novelty is to combine the most general known results on the compactness of Toeplitz and Hankel operators with the standard Fredholm theory to obtain sufficient conditions for $T_{a}$ to be Fredholm.

For a given symbol $a$, the study of the compactness of the Hankel operator $T_{1 / a}$ is closely related with this analysis. We recall the following result of Luecking [7] (see also Theorem 8.35 of [6]; see below for the unexplained notation).

Theorem 1.1. The following conditions are equivalent for all $b \in L^{2}$.

(i) $H_{b}: A^{2} \rightarrow L^{2}$ is compact.

(ii) We have

$$
\left\|f \circ \varphi_{a}-P\left(f \circ \varphi_{a}\right)\right\|_{2} \rightarrow 0 \text { as }|a| \rightarrow 1 .
$$

(iii) The function $F_{b}$ defined by

$$
F_{b}(z)=\inf _{h \in A^{2}} J_{b-h}(z)=\inf _{h \in A^{2}} \frac{1}{|D(z, 1)|} \int_{D(z, 1)}|b-h|^{2} d A
$$

belongs to $C_{0}(\mathbb{D})$, that is, $F_{b}(z) \rightarrow 0$ as $|z| \rightarrow 1$.

(iv) The function $b$ can be decomposed as $b=b_{1}+b_{2}$, where $b_{1} \in C^{1}(\mathbb{D})$ with

$$
\left(1-|z|^{2}\right) \bar{\partial} b_{1}(z) \in C_{0}(\mathbb{D}),
$$

and the function $J_{b_{2}}$ belongs to $C_{0}(\mathbb{D})$.

For bounded symbols, a similar characterization of compact Hankel operators on $A^{2}$ was first given in $[8,9]$, which answered the question raised by Axler, [10]. This was recently extended to all Bergman spaces $A^{p}$ with $1<p<\infty$ in [11], where it was also shown that compactness of Hankel operators with bounded symbols is in fact independent of $p$. See [11] for further details about compact Hankel operators 
with bounded symbols. For other recent works on Hankel operators in Bergman spaces we refer to [12] and [13].

In the next section, we will present a modification of the implication $(i v) \Rightarrow(i)$ of Theorem 1.1, which works for all Bergman spaces $A^{p}$ and all symbols in $L^{1}$. It is worth noting that compactness criteria for $H_{1 / a}$ often involve $V M O_{\delta}$-type conditions (see Definition 1.4 below) and these are usually used to prove Fredholmness of $T_{a}$; see, e.g. $[14,11,15]$, which show that if $a \in L^{\infty} \cap V M O_{\delta}^{1}$, then $T_{a}$ is Fredholm on $A^{p}$ if and only if $\tilde{a}$ is bounded away from zero in some annulus $\{z: r<|z|<1\}$, where $\tilde{a}$ is the Berezin transform of $a$ (see [6] for the definition of $\tilde{a}$ ). As remarked very recently in [16], Fredholmness of $T_{a}$ can be characterized for $a \in B M O_{\delta}^{1}$ provided that $\tilde{a} \in L^{\infty} \cap V O$ by writing $a=\tilde{a}+(a-\tilde{a})$ and observing that $T_{a-\tilde{a}}$ is compact, so that $T_{a}$ is Fredholm if and only if $T_{\tilde{a}}$ is Fredholm; then, one uses the results of [17] on the Fredholm properties of the operator $T_{\tilde{a}}$.

This naturally leads to the question of describing the Fredholm properties of Toeplitz operators with bounded symbols that are not in $V M O_{\delta}^{1}$. In view of the weak condition for the compactness of $T_{a}$ in [5], the requirement of $a$ or $1 / a$ to be in $V M O_{\delta}$ is certainly not necessary for $T_{a}$ to be Fredholm. Finding a complete description remains as a considerable challenge, but we think that Theorem 2.2 and the examples we provide in Section 3 may well be useful for further studies.

The following notation will be used throughout the paper. By $C, C^{\prime}, c$ etc. we denote positive constants, the exact value of which may vary from place to place but not in the same chain of inequalities. If the constant depends on some parameter or function, say, $n$ or $g$, this is denoted by $C_{n}$ or $C_{g}$ etc. All function spaces consist of functions on the disc $\mathbb{D}$, unless otherwise stated. In particular, the space of bounded analytic functions on the disc is denoted by $H^{\infty}$. For clarity, we write $C^{k}(\mathbb{D})$ for the space of $k$ times continuously differentiable functions in $\mathbb{D}$, where $k \in \mathbb{N} \cup\{\infty\}$. The symbol $C_{0}^{k}(\mathbb{D})$ denotes the subspace consisting of functions with compact support in $\mathbb{D} ; k$ is omitted from the notation, if it equals 0 . Given $f \in C^{1}(\mathbb{D})$, we denote the Wirtinger symbols by $\partial=\partial_{x}+i \partial_{y}, \bar{\partial}=\partial_{x}-i \partial_{y}$, where $\partial_{x}=\partial / \partial x, \partial_{y}=\partial / \partial y$ for $z=x+i y$. Also, we use $\partial_{r}=\partial / \partial r$ and $\partial_{\theta}=\partial / \partial \theta$ for the polar coordinates. We denote the Möbius transform by

$$
\varphi_{a}(z)=\frac{a-z}{1-\bar{a} z} \quad \text { for } a, z \in \mathbb{D} .
$$

For $0<\rho<1$ we set $\mathbb{D}_{\rho}:=\{z \in \mathbb{D}:|z| \leq \rho\}$. Given $a \in L^{1}$, we denote by $a_{\rho}$ the function, which coincides with $a$ on $\mathbb{D}_{\rho}$ and equals 0 elsewhere.

We let $\mathcal{D}$ be a family of the sets $D:=D(r, \theta) \subset \mathbb{D}$, where

$$
D=\left\{\rho e^{i \phi} \mid r \leq \rho \leq 1-\frac{1}{2}(1-r), \theta \leq \phi \leq \theta+\pi(1-r)\right\}
$$

for all $0<r<1, \theta \in[0,2 \pi]$. We denote $|D|:=\int_{D} d A$ and, for $\zeta=\rho e^{i \phi} \in D(r, \theta)$,

$$
\hat{a}_{D}(\zeta):=\frac{1}{|D|} \int_{r}^{\rho} \int_{\theta}^{\phi} a\left(\varrho e^{i \varphi}\right) \varrho d \varphi d \varrho .
$$

In the sequel we will consider functions $a \in L^{1}$ such that there exists a constant $C>0$ such that

$$
\left|\hat{a}_{D}(\zeta)\right| \leq C
$$


for all $D \in \mathcal{D}$ and all $\zeta \in D$. The following result is contained in Theorem 2.1 of [18], and it contains the generalized definition of the Toeplitz operator, which we use for symbols satisfying (1.4) and which coincides with the conventional definition whenever the integral (1.1) converges. This generalization of the definition of Toeplitz operators is thus a most natural one, it coincides with the conventional definition whenever the integral formula (1.1) makes sense, and it is considered from many points of view in [18], [19].

Theorem 1.2. Let $1<p<\infty$. If a symbol $a \in L^{1}$ satisfies the condition (1.4), then the limit

$$
T_{a} f=\lim _{\rho \rightarrow 1} T_{a_{\rho}} f, \text { where } T_{a_{\rho}} f(z)=\int_{\mathbb{D}_{\rho}} \frac{a(\zeta) f(\zeta)}{(1-z \bar{\zeta})^{2}} d A(\zeta), \quad f \in A^{p},
$$

converges in the strong operator topology and defines a bounded operator $T_{a}: A^{p} \rightarrow$ $A^{p}$ for all $1<p<\infty$.

Moreover, the transposed operator $T_{a}^{*}: A^{q} \rightarrow A^{q}$ can be written as

$$
T_{a}^{*} f(z)=\lim _{\rho \rightarrow 1} \int_{\mathbb{D}_{\rho}} \frac{\bar{a}(\zeta) f(\zeta)}{(1-z \bar{\zeta})^{2}} d A(\zeta)
$$

for $f \in A^{q}$ and this limit also converges in the strong operator topology.

Notice that for a fixed $0<\rho<1$ the restriction of any $f \in A^{p}$ to $\mathbb{D}_{\rho}$ is a bounded function and the operator $T_{a_{\rho}}$ is bounded in $A^{p}$. Formula (1.5) allows us to define the Toeplitz operator even in many cases, where the defining integral of the conventional formula (1.1) does not converge. In addition, condition (1.4) is also necessary for the boundedness when $a$ is positive. Whether our result gives a complete description of bounded Toeplitz operators with general symbols remains an open problem.

As for the compactness of Toeplitz operators, we have the following result, which is Theorem 2.6 of [5].

Theorem 1.3. Let $p$ and $a$ be as in Theorem 1.2, in particular, a satisfies (1.4). If a has the property

$$
\lim _{d(D) \rightarrow 0} \sup _{\zeta \in D}\left|\hat{a}_{D}(\zeta)\right|=0
$$

where

$$
d(D)=\operatorname{dist}(D, \partial \mathbb{D})=\inf \{|z-w|: z \in D,|w|=1\}
$$

then $T_{a}: A^{p} \rightarrow A^{p}$ is compact.

We next recall the definition of the space $V M O_{\delta}^{p}$. Let us fix $r$ for a moment. The notion of the mean oscillation $M O_{r}^{p}(f)$ of a function $f$ in $L^{p}$ is defined by

$$
M O_{r}^{p}(f)(z)=\left(\frac{1}{|B(z, r)|} \int_{B(z, r)}\left|f(\zeta)-\hat{f}_{r}(z)\right|^{p} d A(\zeta)\right)^{1 / p}
$$

where $B(z, r)$ is the disc with center $z$ and radius $r>0$ in the Bergman metric (see [6], Section 8.1) and

$$
\hat{f}_{r}(z)=\frac{1}{|B(z, r)|} \int_{B(z, r)} f d A
$$

We write $B(z)=B(z, 1)$ and $\hat{f}=\hat{f}_{1}$ in the sequel. 
Definition 1.4. The space of bounded mean oscillation $B M O_{r}^{p}$ consists of all locally $L^{p}$-integrable functions for which $\sup _{z \in \mathbb{D}} M O_{r}^{p}(f)(z)<\infty$. If, in addition, $M O_{r}^{p}(f)(z) \rightarrow 0$ as $|z| \rightarrow 1$, we say that $f$ is in $V M O_{r}^{p}$.

The definition of the spaces is independent of $r$ so that we write $B M O_{\partial}^{p}$ for $B M O_{r}^{p}$ and $V M O_{\partial}^{p}$ for $V M O_{r}^{p}$. Note that $B M O_{r}^{\partial} \subset B M O_{\partial}^{q}$ properly for $q<p$, and $L^{\infty} \cap V M O_{\delta}^{p}=L^{\infty} \cap V M O_{\delta}^{1}$ for all $p>1$.

We finish this section with a short account of Fredholm theory; for a full treatment, see, e.g., Section 3.16 of [20]. A bounded linear operator $T$ on a Banach space $X$ is said to be Fredholm if both its kernel and cokernel are finite dimensional. We note that Fredholm operators have closed range. The index ind $T$ of $T$ is defined to be the difference of the two dimensions.

We need the following characterizations. A bounded linear operator $S$ is called a left parametrix (or a regularizer) of an operator $T$ if $S T=I+K_{1}$ and a right parametrix if $T S=I+K_{2}$ for some compact operators $K_{1}$ and $K_{2}$. It is well known that a bounded linear operator $T$ is Fredholm on $X$ if and only if $T$ has a left and right parametrix. Another useful characterization is that $T$ is Fredholm if and only if the equivalence class $T+K(X)$ is invertible in the Calkin algebra $B(X) / K(X)$, the quotient of the spaces of bounded and compact operators on $X$. Also, we recall that the Fredholm index is stable under small perturbations; that is, for each operator $T \in B(X)$, there is an $\epsilon>0$ such that $T+S$ is Fredholm and $\operatorname{ind}(T+S)=\operatorname{ind} T$, if $S \in B(X)$ and the operator norm satisfies $\|S\|<\epsilon$. Regarding compact perturbations, if $T$ is Fredholm, then $T+K$ is Fredholm and $\operatorname{ind}(T+K)=\operatorname{ind} T$ whenever $K$ is compact. In particular, if $T$ is invertible and $K$ is compact, then $T+K$ is Fredholm of index zero.

\section{Sufficient CONDition for Fredholmness.}

We start by the following generalization of one of the statements of Theorem 1.1. The proof follows the original one, [7], [6], but we have to pay attention to a number of details, because of the more general setting. We expose the details for the convenience of the reader.

Lemma 2.1. Assume that $b \in L^{1} \cap C^{1}(\mathbb{D})$ and that $b$ satisfies the condition (1.4) and

$$
\left(1-|z|^{2}\right) \bar{\partial} b(z) \in C_{0}(\mathbb{D}) .
$$

Then, the Hankel operator $H_{b}: A^{p} \rightarrow L^{p}$ is compact for any $1<p<\infty$.

The definition of the Hankel operator needs to be clarified, since the assumptions do not imply that $M_{b}$ maps $A^{p}$ into $L^{p}$; notice that by assumption the Toeplitz operator $T_{b}$ is bounded in $A^{p}$ at least in the sense of the generalized definition of Theorem 1.2. However, the multiplier maps for example $H^{\infty}$ into $L^{1}$. Thus, arguments simpler than those in (2.3)-(2.5) and (2.11)-(2.16), below, can be used to obtain the norm bound

$$
\left\|H_{b} g\right\|_{p} \leq C\|g\|_{p}
$$

for all $g \in H^{\infty}$ so that the Hankel operator can be defined in the standard way by a bounded extension from the dense subspace $H^{\infty}$ of $A^{p}$. 
Proof. We start by the remark that

$$
\left\langle T_{b} g, \partial h\right\rangle=0 \text {, hence, }\left\langle H_{b} g, \partial h\right\rangle=\left\langle b g-T_{b} g, \partial h\right\rangle=\langle b g, \partial h\rangle
$$

for all $g \in H^{\infty}, b$ as in the assumption of the lemma, and $h \in C_{0}^{\infty}(\mathbb{D})$; note that the duality bracket is certainly well defined since $T_{b} g$ and $b g$ belong to $L^{1}$. Indeed, following the proof of Havin's lemma (Lemma 8.30 in [6]; see in particular the formula above (8.8) there) we find that the Green theorem and the product rule imply for all $\varphi \in C^{1}(\mathbb{D})$

$$
\int_{\mathbb{D}} \bar{\partial}(\varphi \bar{h}) d A=0, \text { hence, } \int_{\mathbb{D}} \varphi \overline{\partial h} d A=-\int_{\mathbb{D}}(\bar{\partial} \varphi) \bar{h} d A \text {. }
$$

In particular,

$$
\int_{\mathbb{D}} T_{b} g \overline{\partial h} d A=-\int_{\mathbb{D}}\left(\bar{\partial} T_{b} g\right) \bar{h} d A
$$

This vanishes, since $T_{b} g$ is analytic, and (2.3) thus holds true.

Second, we note that Havin's lemma holds also in the non-Hilbert setting as follows: we denote by $X$ the closure of the subspace $\left\{\partial \psi: \psi \in C_{0}^{\infty}(\mathbb{D})\right\}$ in $L^{q}$, where $1 / q+1 / p=1$, and claim that

$$
X=Q\left(L^{q}\right)=(I-P)\left(L^{q}\right) .
$$

Indeed, we have for all $f \in L^{p}, \psi \in C_{0}^{\infty}(\mathbb{D})$, by $(2.4)$

$$
\langle f, P \partial \psi\rangle=\langle P f, \partial \psi\rangle=\langle\bar{\partial} P f, \psi\rangle=0 \Rightarrow P \partial \psi=0 \Rightarrow X \subset Q\left(L^{q}\right) .
$$

On the other hand, if $Q\left(L^{q}\right) \backslash X \neq\{0\}$, we fix a function $f \in Q\left(L^{q}\right) \backslash X, f \neq 0$ and apply the Hahn-Banach theorem in the dual pair of $L^{p}$ and $L^{q}$ to find a function $g \in L^{p}, g \neq 0$, such that

$$
\langle g, f\rangle \neq 0 \text { and } 0=\langle g, \partial \psi\rangle=\langle\bar{\partial} g, \psi\rangle \forall \psi \in C_{0}^{\infty} .
$$

But these imply

$$
\langle Q g, f\rangle=\langle g, Q f\rangle=\langle g, f\rangle \neq 0
$$

and, since $X \subset Q\left(L^{q}\right)$,

$$
\langle\bar{\partial}(Q g), \psi\rangle=\langle Q g, \partial \psi\rangle=\langle g, Q(\partial \psi)\rangle=\langle g, \partial \psi\rangle=0 \forall \psi \in C_{0}^{\infty} .
$$

so that $Q g \neq 0$ and $\bar{\partial}(Q g)=0$, hence, $Q g$ is a non-zero analytic function, which is a contradiction. Thus (2.6) holds.

We will need the following consequence of (2.6): for all $g \in L^{p}$ we obtain

$$
\begin{gathered}
\|Q g\|_{p}=\sup _{\substack{\tilde{h} \in L^{q},\|\tilde{h}\|_{q} \leq 1}}\langle Q g, \tilde{h}\rangle=\sup _{\substack{\tilde{h} \in L^{q},\|\tilde{h}\|_{q} \leq 1}}\left\langle Q^{2} g, \tilde{h}\right\rangle=\sup _{\substack{\tilde{h} \in L^{q},\|\tilde{h}\|_{q} \leq 1}}\langle Q g, Q \tilde{h}\rangle \\
\leq \sup _{\substack{f \in X \\
\|f\|_{q} \leq 1}}\langle Q g, f\rangle=\sup _{\substack{\tilde{f} \in L^{q},\|\partial \tilde{f}\|_{q} \leq 1}}\langle Q g, \partial \tilde{f}\rangle,
\end{gathered}
$$

where we used the fact that $\|Q \varphi\|_{q} \leq C\|\varphi\|_{q}$ for all $\varphi \in L^{q}$, since $Q$ is a bounded projection.

We consider a sequence $\left(g_{n}\right)_{n=1}^{\infty} \subset A^{p}$, which is bounded in the norm of $L^{p}$ and converges to 0 uniformly on compact subsets of $\mathbb{D}$. It suffices to show that $H_{b}$ maps 
this sequence into one converging to 0 in the norm of $L^{p}$. First, we choose for all $n$ a polynomial $\tilde{g}_{n}$ such that

$$
\left\|g_{n}-\tilde{g}_{n}\right\|_{p} \leq \frac{1}{n}
$$

Since $T_{b}$ is a bounded operator by assumption, $T_{b} g_{n} \rightarrow 0$ as $n \rightarrow \infty$ in $A^{p}$ if and only if $T_{b} \tilde{g}_{n} \rightarrow 0$ in $A^{p}$. Also, the polynomials $\tilde{g}_{n}$ converge to 0 uniformly on bounded sets and certainly also form a bounded set, as a consequence of (2.10). Thus, we may assume from the very beginning that the original sequence consists of polynomials, which in particular are members of $H^{\infty} \subset A^{2}$.

Given an arbitrary $\varepsilon>0$ we fix a compact set $K$ such that

$$
\left(1-|z|^{2}\right)|\bar{\partial} b(z)|<\varepsilon \text { for all } z \in \mathbb{D} \backslash K
$$

and then $N \in \mathbb{N}$ such that

$$
\left|g_{n}(z)\right| \leq \frac{\varepsilon}{1+\sup _{z \in K}|\bar{\partial} b(z)|} \text { for all } z \in K, n \geq N .
$$

We write for all $h \in \mathbb{C}_{0}^{\infty}$, using (2.3) and (2.4),

$$
\begin{aligned}
& \left\langle H_{b} g_{n}, \partial h\right\rangle=\left\langle b g_{n}, \partial h\right\rangle=\int_{\mathbb{D}} b g_{n} \overline{\partial h} d A \\
= & -\int_{\mathbb{D}}\left(\bar{\partial}\left(b g_{n}\right)\right) \bar{h} d A=-\int_{\mathbb{D}}(\bar{\partial} b) g_{n} \bar{h} d A .
\end{aligned}
$$

Hence, by the Hölder inequality with $1 / p+1 / q=1$

$$
\begin{aligned}
& \left|\left\langle H_{b} g_{n}, \partial h\right\rangle\right| \\
\leq & \left(\int_{\mathbb{D}}\left(1-|z|^{2}\right)^{p}|\bar{\partial} b(z)|^{p}\left|g_{n}(z)\right|^{p} d A(z)\right)^{1 / p}\left\|\frac{h(z)}{1-|z|^{2}}\right\|_{q}
\end{aligned}
$$

Here we recall that $|\nabla h(z)|^{2}=2\left(|\partial h(z)|^{2}+|\bar{\partial} h(z)|^{2}\right.$ for all $z \in \mathbb{D}$ and thus obtain by Lemmas 8.31 (or its straightforward generalization) and 8.32 of [6]

$$
\left\|\frac{h(z)}{1-|z|^{2}}\right\|_{q} \leq C\|\partial h\|_{q}
$$

Moreover, we divide the $L^{p}$-integration in (2.14) into integrals over $K$ and its complement, and obtain by (2.11), (2.12) for $n \geq N$ the bound

$$
\begin{aligned}
& \int_{\mathbb{D}}\left(1-|z|^{2}\right)^{p}|\bar{\partial} b(z)|^{p}\left|g_{n}(z)\right|^{p} d A(z) \\
\leq & \sup _{z \in K}|\bar{\partial} b(z)|\left|g_{n}(z)\right|+\int_{\mathbb{D} \backslash K}\left(\sup _{w \in \mathbb{D} \backslash K}\left(1-|w|^{2}\right)^{p}|\bar{\partial} b(w)|^{p}\right)\left|g_{n}(z)\right|^{p} d A(z)
\end{aligned}
$$

Combining this with (2.14), (2.15) and taking into account (2.9) yield $\left\|H_{b} g_{n}\right\|_{p} \leq C \varepsilon$, which was to be proven.

The following is our main result on the Fredholm properties of Toeplitz operators. As for the calculation of the Fredholm index, we comment it only in the examples in Section 3. 
Theorem 2.2. Assume that the symbol $a \in L^{1}$ has property (1.4) and that there is a function $b \in L^{1}$ satisfying (1.4) such that

$1^{\circ}$. $b$ and $\bar{b}$ satisfy $(2.1)$,

$2^{\circ}$. there exists $\varphi \in L^{1}$ with property (1.4) such that $T_{\varphi}$ is invertible in $A^{p}$ and the symbol $\varphi-a b$ satisfies (1.7), and

$3^{\circ}$. the multipliers $M_{b}$ and $M_{\bar{b}}$ are bounded from below as operators $A^{p} \rightarrow L^{p}$.

Then, the operator $T_{a}$ is Fredholm.

Proof. In order to show that $T_{b} T_{\varphi}^{-1}$ is a right parametrix for $T_{a}$ we write

$$
T_{a} T_{b}=T_{\varphi}-T_{\varphi-a b}-P M_{a} H_{b} .
$$

Here, the Toeplitz operator $T_{\varphi-a b}$ is compact by Theorem 1.3. By Lemma 2.1, the Hankel operator $H_{b}: A^{p} \rightarrow L^{p}$ is compact. To prove that $P M_{a} H_{b}$ is compact we need to show that $P M_{a}$ is bounded in $H_{b}\left(A^{p}\right)$; this does not automatically follow from the boundedness of $T_{a}$ since the domain of the operator is different from $A^{p}$. Now, for all $g \in M_{b}\left(A^{p}\right)$ we take $f \in A^{p}$ such that $g=b f$ and write

$$
\begin{aligned}
& \quad\left\|P M_{a} g\right\|_{p}=\left\|P M_{a b} f\right\|_{p}=\left\|P M_{\varphi} f-P M_{\varphi-a b} f\right\|_{p} \\
& \leq\left\|T_{\varphi} f\right\|_{p}+\left\|T_{\varphi-a b} f\right\|_{p} \leq C\|f\|_{p},
\end{aligned}
$$

by the assumption $2^{\circ}$ and Theorem 1.3. Since $M_{b}$ is bounded from below by the assumption $3^{\circ}$, we have $C\|f\|_{p} \leq C^{\prime}\|g\|_{p}$ so that in view of $(2.18), P M_{a}: H_{b}\left(A^{p}\right) \rightarrow$ $A^{p}$ is bounded. Thus $P M_{a} H_{b}$ is a compact operator and $T_{b} T_{\varphi}^{-1}$ is a right parametrix, since

$$
T_{a} T_{b} T_{\varphi}^{-1}=I-T_{\varphi-a b} T_{\varphi}^{-1}-P M_{a} H_{b} T_{\varphi}^{-1} .
$$

where the last two terms are compact operators by what was proven above.

As for the left parametrix, we recall that the adjoint $T_{a}^{*}: A^{q} \rightarrow A^{q}$ of $T_{a}$ coincides with $T_{\bar{a}}$, see Theorem 1.2 and [6], Proposition 7.1. By the assumptions of our proposition and the proof above, we find that

$$
T_{\bar{\varphi}}-T_{\bar{a}} T_{\bar{b}}: A^{q} \rightarrow A^{q}
$$

is a compact operator, where $1 / p+1 / q=1$. (Notice that a function satisfies condition (1.7) if and only if its complex conjugate does.) Hence, by Schauder's theorem, also the operator

$$
\left(T_{\bar{\varphi}}-T_{\bar{a}} T_{\bar{b}}\right)^{*}=T_{\bar{\varphi}}^{*}-T_{\bar{b}}^{*} T_{\bar{a}}^{*}=T_{\varphi}-T_{b} T_{a}: A^{p} \rightarrow A^{p}
$$

is compact so that $T_{\varphi}^{-1} T_{b}$ is a left parametrix. Thus, $T_{a}$ is Fredholm.

\section{EXAMPLES.}

Let us consider some examples Fredholm Toeplitz operators $T_{a}$. Since the Fredholm property is connected with the compactness of Hankel operators and the latter is related with symbols belonging to the space $V M O_{\delta}$, it is of interest to consider bounded symbols $a \notin V M O_{\delta}$. We start by constructing concrete examples of such symbols. Let us set

$$
\Theta(z)=\operatorname{sgn}(\sin (1 /(1-r)))
$$

where $z=r e^{i \theta} \in \mathbb{D}$ and we denote for all real numbers $s$,

$$
\operatorname{sgn}(s)=\left\{\begin{aligned}
1, & \text { if } s \geq 0 \\
-1, & \text { if } s<0 .
\end{aligned}\right.
$$


The proof in Remark 2.4 of [5] shows that $\Theta$ satisfies property (1.7). We present the details of this proof, since modifications of it will soon be needed. Let $D=$ $D(1-2 \delta, \theta)$ be as in (1.3) for some $\theta \in[0,2 \pi)$ and small enough $\delta>0$ and consider $\zeta=\rho e^{i \phi} \in D$. Using the change of variable $y=1 /(1-\varrho)$ we get

$$
2|D|\left|\hat{\Theta}_{D}(\zeta)\right|=\int_{\theta}^{\phi}\left|\int_{1-2 \delta}^{\rho} \operatorname{sgn}(\sin (1 /(1-\varrho))) d \varrho\right| \leq \pi \delta\left|\int_{1 /(2 \delta)}^{1 /(1-\rho)} \frac{1}{y^{2}} \operatorname{sgn}(\sin y) d y\right|
$$

We divide the integration interval to the subintervals $J_{n}:=[2 \pi n, 2 \pi(n+1)], n \in \mathbb{N}$. We have

$$
\left|\int_{J_{n}} \frac{1}{y^{2}} \operatorname{sgn}(\sin y) d y\right|=\left|\int_{2 \pi n}^{2 \pi n+\pi}\left(\frac{1}{y^{2}}-\frac{1}{(y+\pi)^{2}}\right) d y\right| \leq C \int_{2 \pi n}^{2 \pi n+\pi} \frac{1}{y^{3}} d y \leq C^{\prime} \frac{1}{n^{3}}
$$

which yields

$$
\left|\int_{2 \delta}^{1 /(1-\rho)} \frac{1}{y^{2}} \operatorname{sgn}(\sin y) d y\right| \leq C \sum_{n=[1 /(2 \delta)]}^{\infty} \frac{1}{n^{3}} \leq C^{\prime} \delta^{2}
$$

where $[s]$ denotes the largest integer not bigger than $s \in \mathbb{R}$. We obtain (1.7) by taking into account (3.3) and that $|D|$ is of order $\delta^{2}$.

We show that $\Theta \notin V M O_{\delta}$. Indeed, fixing $r>0$ a calculation similar to (3.3)-(3.4) shows that the average $\hat{\Theta}_{r}$ of $\Theta$ over a set $B(z, r)$ has the bound $\left|\hat{\Theta}_{r}\right| \leq C \delta$, where $\delta=1-|z|$ for all $z \in \mathbb{D}$. On the other hand, we have $|\Theta(z)|=1$ for all $z \in \mathbb{D}$ by the definition, hence, the quantity (1.8) has, for all $z$ close enough to the unit circle, the lower bound

$$
\begin{aligned}
& M O_{r}^{p}(\Theta)(z) \geq\left(\frac{1}{|B(z, r)|} \int_{B(z, r)}\left(|\Theta(\zeta)|-\left|\hat{\Theta}_{r}\right|\right)^{p} d A(\zeta)\right)^{1 / p} \\
\geq & C^{\prime}\left(\frac{1}{|B(z, r)|} \int_{B(z, r)}(1-C \delta)^{p} d A(\zeta)\right)^{1 / p} \geq C^{\prime \prime} .
\end{aligned}
$$

Thus, $\Theta$ belongs to $B M O_{\delta}$ but not to $V M O_{\delta}$.

a) Consider the Toeplitz operator $T_{a}$ with the bounded symbol

$$
a=1+\Theta \text {. }
$$

By the argument above and Theorem 1.3, $T_{\Theta}$ is compact in any space $A^{p}$ so that $T_{a}$ is Fredholm although $a \notin V M O_{\delta}$. Moreover, given any neighborhood $U \subset \overline{\mathbb{D}}$ of the boundary $\partial \mathbb{D}$, the symbol $a$ vanishes in a subset of $U$ with positive area.

More generally, assume that a function $f \in V M O_{\delta}$ satisfies (1.4) and in addition $\left|\hat{f}_{D}(\zeta)\right| \geq C>0$ for all $D \in \mathcal{D}$ and $\zeta \in D$. (Trivial example: $f$ is continuous, real and bounded from below by a positive constant on the closed unit disc.) The operator $T_{a}$ with

$$
a=f+\Theta
$$

is Fredholm, although $a \notin V M O_{\delta}$. See Theorem 2.8 of [5]. The index of $T_{a}$ is of course the same as that of $T_{f}$. 
b) Obviously, the example a) can be modified by endlessly many ways. One could make $\Theta$ a little more slowly oscillating by replacing in (3.1) the function $1 /(1-r)$ by $(1-r)^{-\alpha}$ for any constant $0<\alpha<1$. It could also be made unbounded by multiplying $\Theta$ with $(1-r)^{-\beta}$, for any $0<\beta<1$. Also, the symbol $\Theta$ of (3.1) is not smooth, but it can be written as the sum

$$
\Theta=\Theta_{1}+\Theta_{2}
$$

where $\Theta_{1}$ is an infinitely smooth bounded function, a mollification of $\Theta$, and $\Theta_{2}$ induces a compact Toeplitz operator. Indeed, we set

$$
\Theta_{1}(z)=\int_{\mathbb{C}} J_{z}(w-z) \Theta(w) d A(w),
$$

where $J_{z}, z \in \mathbb{D}$, is for example the scaled mollifier

$$
J_{z}(w)=\nu(z)^{2} J(\nu(z) w) \text { and } \nu(z)=e^{1 /\left(1-|z|^{2}\right)}>1
$$

and $J$ is the standard $C^{\infty}$-mollifier

$$
J(w)= \begin{cases}C e^{-1 /\left(1-|w|^{2}\right)}, & w \in \mathbb{D}, \\ 0, & w \in \mathbb{C},|w| \geq 1\end{cases}
$$

with a normalization factor $C>0$ such that $\int_{\mathbb{C}} J d A=1$. We have $\left|\Theta_{2}(z)\right| \leq 1$ for all $z$. The support of $\Theta_{2}$ is contained in the set

$$
E=\left\{z=r e^{i \theta} \in \mathbb{D}:\left|r-r_{n}\right| \leq 1 / \nu\left(r_{n}\right)=e^{-\pi n} \text { for some } n \in \mathbb{N}\right\}
$$

where $r_{n}=1-1 /(\pi n), n \in \mathbb{N}$, are the discontinuity points of the function $\operatorname{sgn}(\sin (1 /(1-$ $r)$ ), see (3.1). Thus, for any $D \in \mathcal{D}$, the area of a set $E \cap D$ is "small" in comparison with $|D|$, and one can use this to show that $\Theta_{2}$ satisfies (1.7). Hence, $T_{\Theta_{2}}$ and $T_{\Theta_{1}}=T_{\Theta}-T_{\Theta_{2}}$ are compact and

$$
T_{1-\Theta_{1}} \text { and } T_{f-\Theta_{1}}
$$

with $f$ as in the example a), are Fredholm; also, $\Theta_{1} \notin V M O_{\delta}$. We leave it to the reader to verify the details of these claims.

The next example is the most relevant as regards to Theorem 2.2.

c) Let the symbol $\Theta$ with property (1.7) be as above, and let $f \in C^{1}(\mathbb{D})$ be a real function satisfying (2.1) such that

$$
C \leq f(z) \leq C_{1}
$$

for some constants $C_{1}>C>0$ and all $z \in \mathbb{D}$. In view of Theorem 2.2 , the symbol

$$
a=1-f \Theta
$$

induces a Fredholm Toeplitz operator, since for $b=1 / f$ we have $a b=1 / f-\Theta$ and all conditions of the proposition are satisfied; notice that $\bar{\partial} b=(\bar{\partial} f) / f^{2}$.

Apparently, more general functions $f$ and $\Theta$ could be used here, once $\Theta$ has property (1.7) and the function $b=1 / f$ satisfies $1^{\circ}$ and $3^{\circ}$ of Theorem 2.2 and the operator $T_{1 / f}$ is invertible. We do not know if $f \Theta$ has property (1.7) for all such $f$ and $\Theta$. We refer to Proposition 2.2 of [19], where the product of a function with property (1.4) and another function has been studied. 
d) Let us finally consider the symbol

$$
a=\frac{1}{i+\Theta / 2}
$$

where $\Theta$ is as in (3.1). We indicate two ways to show that $T_{a}: A^{p} \rightarrow A^{p}$ is Fredholm for all $p, 1<p<\infty$. First, let us define $b(z)=5 i / 4$ so that $1^{\circ}$ of Theorem 2.2 trivially holds. Moreover, we have $\Theta(z)^{2}=1 / 4$ and thus

$$
\sum_{n=0}^{\infty} \tilde{a}(z)^{4 n}=\frac{16}{15}
$$

for all $z \in \mathbb{D}$, hence

$$
\begin{aligned}
a(z) b(z) & =\frac{5}{4} \frac{1}{1-i \Theta}=\frac{5}{4} \sum_{n=0}^{\infty}(i \Theta)^{n}=\frac{5}{4}\left(\sum_{n=0}^{\infty} \tilde{a}^{4 n}+i \Theta^{4 n+1}-\Theta^{4 n+2}-i \Theta^{4 n+3}\right) \\
10) & =\frac{5}{4}\left(\frac{16}{15}-\frac{16}{4 \cdot 15}\right)+\frac{5 i}{4} \Theta\left(\frac{16}{2 \cdot 15}-\frac{16}{8 \cdot 15}\right)=1+\Theta \frac{i}{2}
\end{aligned}
$$

As conclusion, property $2^{\circ}$ holds, by what was remarked on the symbol $\Theta$. As for $3^{\circ}$, this trivially holds for $M_{b}$ and $M_{\bar{b}}$.

On the other hand one can also use a calculation similar to (3.10) to show directly that the symbol $a$ can be written as $\alpha+\beta \Theta$ for some constants $\alpha, \beta \in \mathbb{C} \backslash\{0\}$ so that the Fredholm property follows in the same way as in the example a).

\section{Conclusions.}

In the main general Theorem 2.2 we have combined an existing "weak" condition on the compactness of Toeplitz operators with an improved result on the compactness of Hankel operators, to get a new, weak condition for the Fredholm property of a given Toeplitz operator $T_{a}$. According to the basic intuition, for a very regular $a$, like a continuous one on the closed unit disc $\overline{\mathbb{D}}$, the symbol must be non-zero on the boundary, in order to induce a Fredholm Toeplitz operator. Passing to more general symbols $a$, it is a consequence of our main theorem that there are cases where, for any neighborhood $U$ of the boundary $\partial \mathbb{D}$, a may vanish in a subset of $U$ with positive measure. This counterintuitive result is explained by the cancellation phenomena, which according to the proof of the main theorem and the reference [5] may lead to compact Toeplitz operators.

Our theorem also leads to many examples of Fredholm Toeplitz operators, where the assumption on the $V M O_{\delta}$-property of the symbol can be relaxed, although this condition often appears in the existing literature.

\section{REFERENCES}

[1] Englis M. An excursion into Berezin-Toeplitz quantization and related topics, in: Quantization, PDEs, and Geometry (D. Bahns, W. Bauer, I. Witt, editors), Operator Theory Advances and Applications. Basel, Switzerland: Birkhäuser; 2016, 69-11.

[2] Fedosov B. Deformation Quantization and Index Theory. New Your, USA: Akademie Verlag; 1995.

[3] Grudsky S, Rybkin A. On Toeplitz and Hankel operators with oscillatory symbols containing Blaschke products and applications to the $\mathrm{KdV}$ equation. Operator theory, pseudodifferential equations, and mathematical physics,Oper. Theory Adv. Appl. 228. Basel, Switzerland: Birkhäuser/Springer Basel AG; 2013, 127-150. 
[4] Grudsky S, Rybkin A. Soliton thoery and Hankel operators. Siam J. Math. Anal 2015; 47 (5): 2283-2323

[5] Taskinen J, Virtanen J. Toeplitz operators on Bergman spaces with locally integrable symbols. Rev. Mat. Iberoam. 2010; 26 (2): 693-706.

[6] Zhu K. Operator Theory in Function Spaces, 2nd edition, Mathematical Surveys and Monographs 138. Providence, RI, USA: American Mathematical Society; 2007.

[7] Luecking D. Characterizations of certain classes of Hankel operators on the Bergman spaces of the unit disk. J. Funct. Anal. 1992; 110 (2): 247-271.

[8] Stroethoff K. Compact Hankel operators on the Bergman space. Illinois J. Math. $1990 ; 34$ (1): 159-174.

[9] Zheng D. Toeplitz operators and Hankel operators. Integral Equations Operator Theory 1989; 12 (2): 280-299.

[10] Axler S. The Bergman space, the Bloch space, and commutators of multiplication operators. Duke Math. J. 1986; 53 (2): 315-332.

[11] Hagger R, Virtanen J.A. Compact Hankel operators with bounded symbols. arXiv:1906.09901.

[12] Peláez J, Perälä A, Rättyä J. Hankel operators induced by radial Bekollé-Bonami weights on Bergman spaces. arXiv:1806.09854

[13] Hu Z, Lu J. Hankel Operators on Bergman Spaces with Regular Weights. J. Geom. Analysis 2018.

[14] Hagger R. The essential spectrum of Toeplitz operators on the unit ball. Integral Equations Operator Theory 2017; 89 (4): 519-556.

[15] Perälä A, Virtanen J.A. A note on the Fredholm properties of Toeplitz operators on weighted Bergman spaces with matrix-valued symbols. Oper. Matrices 2011; 5 (1): 97-106.

[16] Zorboska N. Closed range type properties of Toeplitz operators on the Bergman space and the Berezin transform. Complex Anal. Oper. Theory 2019; 13 (8): 4027-4044.

[17] Stroethoff K, Zheng D. Toeplitz and Hankel operators on Bergman spaces, Trans. Amer. Math. Soc. 1992; 329 (2): 773-794.

[18] Taskinen J, Virtanen J.A. On generalized Toeplitz and little Hankel operators on Bergman spaces. Arch. Math. 2018; 110 (2): 155-166.

[19] Taskinen J, Virtanen J.A. On compactness of Toeplitz operators in Bergman spaces. Funct. Approx. Comment. Math. 2018; 59 (2): 305-318.

[20] Simon B. Operator theory. A Comprehensive Course in Analysis, Part 4. Providence, RI, USA: American Mathematical Society; 2015.

Department of Mathematics and Statistics, University of Helsinki, P.O. Box 68, 00014 HeLsinki, Finland

E-mail address: jari.taskinen@helsinki.fi

Department of Mathematics, University of Reading, Whiteknights, P.O. Box 220, READING RG6 6AX, UK

E-mail address: j.a.virtanen@reading.ac.uk 